\title{
The novel E-subgroup pentatricopeptide repeat protein DEK55 is responsible for RNA editing at multiple sites and for the splicing of nad 1 and nad4 in maize
}

Ru Chang Ren ${ }^{1 \dagger}$, Xu Wei Yan ${ }^{1 \dagger}$, Ya Jie Zhao ${ }^{1+}$, Yi Ming Wei ${ }^{1}$, Xiaoduo $L u^{2}$, Jie Zang ${ }^{1}$, Jia Wen Wu', Guang Ming Zheng ${ }^{1}$, Xin Hua Ding ${ }^{3}$, Xian Sheng Zhang ${ }^{1}$ and Xiang Yu Zhao ${ }^{1 *}$ (D)

\begin{abstract}
Background: Pentatricopeptide repeat (PPR) proteins compose a large protein family whose members are involved in both RNA processing in organelles and plant growth. Previous reports have shown that E-subgroup PPR proteins are involved in RNA editing. However, the additional functions and roles of the E-subgroup PPR proteins are unknown.

Results: In this study, we developed and identified a new maize kernel mutant with arrested embryo and endosperm development, i.e., defective kernel (dek) 55 (dek55). Genetic and molecular evidence suggested that the defective kernels resulted from a mononucleotide alteration (C to $T$ ) at $+449 \mathrm{bp}$ within the open reading frame (ORF) of Zm00001d014471 (hereafter referred to as DEK55). DEK55 encodes an E-subgroup PPR protein within the mitochondria. Molecular analyses showed that the editing percentage of 24 RNA editing sites decreased and that of seven RNA editing sites increased in dek55 kernels, the sites of which were distributed across 14 mitochondrial gene transcripts. Moreover, the splicing efficiency of nad 1 introns 1 and 4 and nad 4 intron 1 significantly decreased in dek55 compared with the wild type (WT). These results indicate that DEK55 plays a crucial role in RNA editing at multiple sites as well as in the splicing of nad 1 and nad4 introns. Mutation in the DEK55 gene led to the dysfunction of mitochondrial complex I. Moreover, yeast two-hybrid assays showed that DEK55 interacts with two multiple organellar RNA-editing factors (MORFs), i.e., ZmMORF1 (Zm00001d049043) and ZmMORF8 (Zm00001d048291).
\end{abstract}

Conclusions: Our results demonstrated that a mutation in the DEK55 gene affects the mitochondrial function essential for maize kernel development. Our results also provide novel insight into the molecular functions of Esubgroup PPR proteins involved in plant organellar RNA processing.

Keywords: Defective kernel, Maize, Mitochondrion, Pentatricopeptide repeat proteins, RNA processing, Splicing

\footnotetext{
* Correspondence: zhxy@sdau.edu.cn

${ }^{\dagger}$ Ru Chang Ren, Xu Wei Yan and Ya Jie Zhao contributed equally to this work.

${ }^{1}$ State Key Laboratory of Crop Biology, College of Life Sciences, Shandong Agricultural University, Taian, Shandong 271018, PR China

Full list of author information is available at the end of the article
}

(c) The Author(s). 2020 Open Access This article is licensed under a Creative Commons Attribution 4.0 International License, which permits use, sharing, adaptation, distribution and reproduction in any medium or format, as long as you give appropriate credit to the original author(s) and the source, provide a link to the Creative Commons licence, and indicate if changes were made. The images or other third party material in this article are included in the article's Creative Commons licence, unless indicated otherwise in a credit line to the material. If material is not included in the article's Creative Commons licence and your intended use is not permitted by statutory regulation or exceeds the permitted use, you will need to obtain permission directly from the copyright holder. To view a copy of this licence, visit http://creativecommons.org/licenses/by/4.0/ The Creative Commons Public Domain Dedication waiver (http://creativecommons.org/publicdomain/zero/1.0/) applies to the data made available in this article, unless otherwise stated in a credit line to the data. 


\section{Background}

Pentatricopeptide repeat (PPR) proteins compose a large protein family found in most land plants, with more than 450 members identified in Arabidopsis thaliana, Oryza sativa, and Zea mays [1-5]. These proteins contain standard tandem degenerate repeat motifs, which form a helix-loop-helix structure of approximately 35 amino acids. PPR proteins are classified mainly into Pand PLS-type subfamilies according to their PPR repeat motifs $[2,6,7]$. P-subfamily PPR proteins contain only classic "P" motif repeats in tandem, while PLS-subfamily PPR proteins contain alternating repeats of three PPR motifs of different lengths. The latter are usually divided into PLS, E, E+, and DYW subgroups based on the presence of $\mathrm{E}, \mathrm{E}+$, or DYW domains at the carboxy-terminal end [2]. A new class of PPR proteins that contain small MutS-related domains at the carboxy-terminal end has also been identified, $[8,9]$.

P-type PPRs are considered to be involved in group II intron splicing, RNA stabilization, cleavage, translational activation, and transcript accumulation. In contrast, PLStype PPRs play essential roles in the conversion of cytidine (C) to uridine $(\mathrm{U})$ at specific sites of organelle transcripts $[4,10,11]$. Most plant PPR proteins are targeted to mitochondria, chloroplasts, or both and regulate the functions and development of those organelles [10]. In the mitochondria, the oxidative phosphorylation system comprises five complexes (I-V) [12]. Normal assembly of these complexes is essential to maintain mitochondrial function, which requires the standard processing of mitochondrial pre-mRNAs, involving both RNA editing and intron splicing $[13,14]$. Numerous PPRs are responsible for RNA posttranscriptional processes in mitochondria [15-21].

E-subgroup PPR proteins (e.g., slow growth 1 (SLO1), organelle transcript processing 87 (OTP87), mitochondrial editing factor 3 (MEF3), MEF9, MEF12, and mitochondrial PPR 25 (MPR25)) play vital roles in mitochondrial RNA editing and plant development [2227]. In addition, several E-subgroup proteins in Arabidopsis and rice are involved in RNA splicing [28, 29]. In maize, five E-subgroup PPR proteins have been characterized, and all of them are involved in RNA editing. SMALL KERNEL (SMK) 1 (SMK1) is critical for nad7836 editing in mitochondria and is conserved in maize and rice [14]. SMK4 is critical for RNA editing of cytochrome $c$ oxidase 1 (cox 1$)$ at position +1489 bp [30], $c c m F_{N}$ is essential for cytochrome c maturation, and EMPTY PERICARP 7 (EMP7) is responsible for its editing at the $+1553 \mathrm{bp}$ position [31]. DEK39 is necessary for RNA editing of nad3 transcripts in mitochondria [32], and Dek10 is responsible for RNA editing of three sites of nad3 and cox 2 transcripts [33]. However, it is still unclear whether E-subgroup PPRs are involved in RNA editing and intron splicing in maize organelles.
Here, we identified maize mutant dek55, which has an embryo-lethal phenotype and arrested endosperm development, which is caused by a mutation of the mitochondrion-localized E-subgroup PPR protein DEK55. In the dek55 mutant, the splicing efficiency of nad1 introns 1 and 4 trans-splicing and nad4 intron 1 cis-splicing decreased. Moreover, the editing percentages of 24 editing sites (atp1-1490, atp8-123, ccmFc-160, ccmFc-799, ccmFc-866, ccmFc-906, ccmFc-1144, ccmFc1244, ccmFn-287, ccmFn-302, cob-564, mat-r-1877, nad3-146, nad3-190, nad4-77, nad6-25, nad6-138, nad6-146, nad6-159, nad6-161, rps12-ct-418, rps12284, ribosomal protein S13 (rps13)-56, and rps3-69) was also substantially reduced. Further, DEK55 could interact with ZmMORF1 and ZmMORF8 in yeast, which might be responsible for the activity of DEK55 on multiple editing sites. Taken together, our results suggest that the E-subgroup PPR protein DEK55 is involved in both RNA editing and group II intron splicing in maize mitochondria.

\section{Results}

Genetic and phenotypic analysis of the defective kernel 55-1 (dek55-1) mutant

A mutant with a defective kernel phenotype was isolated from an ethylmethanesulfonate-induced maize B73 background population and was subsequently named dek55-1. The dek55-1 kernels segregated from selfpollinated progeny of dek55-1/+ heterozygotes at a 1:3 mendelian ratio (Fig. 1a, Additional file 1: Table S1). These results suggested that dek55-1, which exhibits a recessive phenotype, is caused by a monogenic mutation, which was confirmed in other populations generated from dek55-1/+ heterozygotes crossed with C733 or S162 inbred lines (Additional file 1: Table S1).

The dek55-1 kernels were smaller and presented a whitish pericarp, and they could be distinguished from the wild-type (WT) kernels at 15 days after pollination (DAP) (Fig. 1a). At maturity, the dek55-1 kernels were much smaller and shrivelled (Fig. 1b, c). To further determine the mutant phenotype, both WT and dek55-1 kernels were longitudinally sliced at 15 DAP. Compared to the WT kernels, the mutant kernels had a small, soft endosperm. (Fig. 1d, e). Furthermore, compared with the WT kernels, the dek55-1 kernels contained a smaller mature embryo and a reduced proportion of hard endosperm (Fig. 1f, g). In addition, the weight of dek55-1 kernel was approximately $70 \%$ lower than that of WT kernels (Fig. 1h). No dek55-1 seeds (0/100) germinated under field conditions, implying that embryo arrest is lethal in the mutants.

To further investigate the developmental structure of dek55-1 kernels, we examined the kernel tissue structure of the WT and dek55-1 mutant at 12 and 18 DAP 

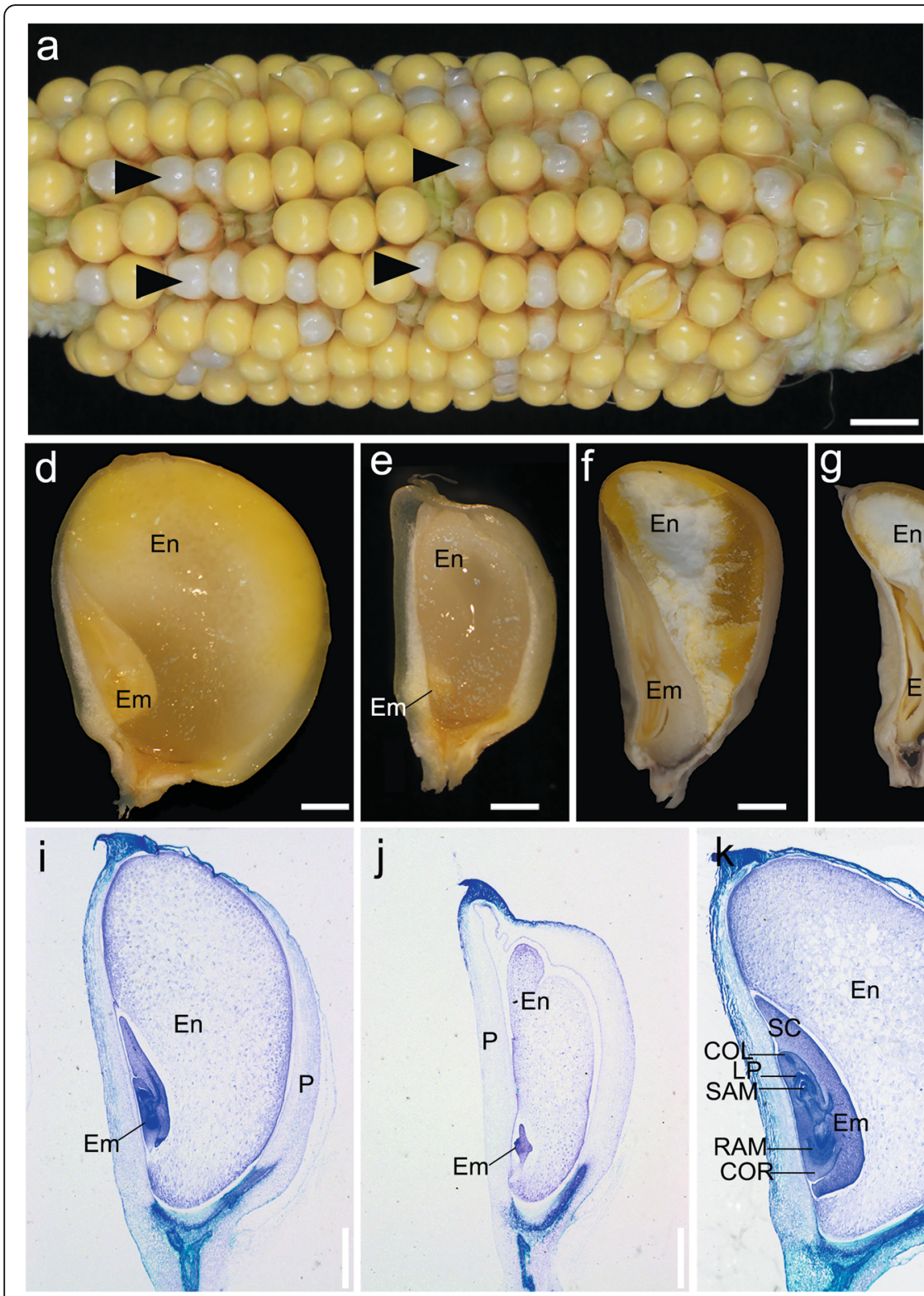
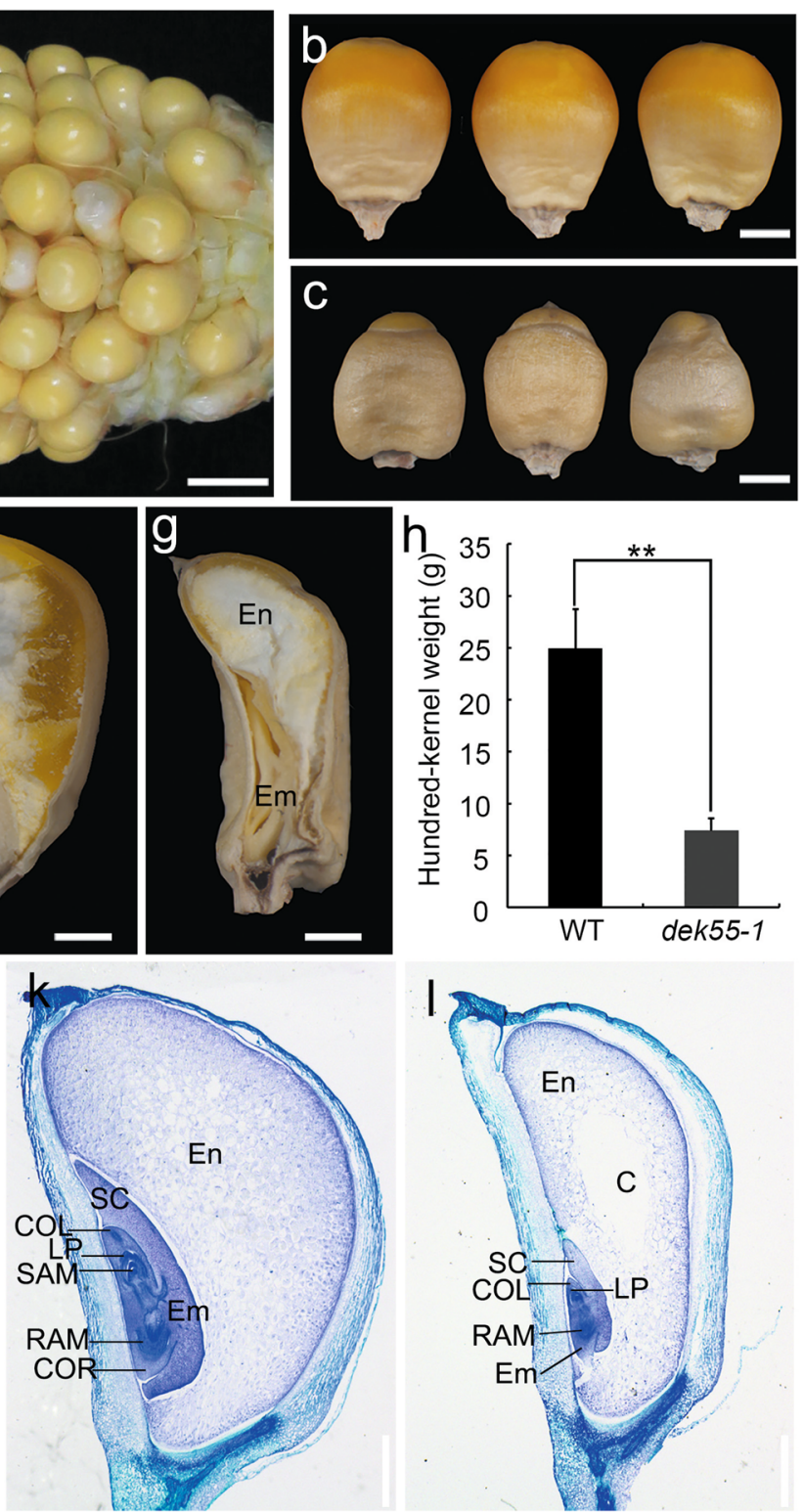

Fig. 1 Phenotypic characterization of dek55-1 kernels. a Self-pollinated dek55-1 heterozygous ears at 15 DAP. Several mutant kernels are indicated with arrowheads. Scale bars $=1 \mathrm{~cm}$. b-c Mature kernels of WT and dek55-1 plants. b. WT. c, dek55-1. Scale bars $=2 \mathrm{~mm}$. $\mathbf{d}-\mathbf{g}$ Comparative anatomy of WT and dek55-1 kernels at 15 DAP and at maturity. $\mathbf{d}$ and $\mathbf{f}$ WT kernels. e and $\mathbf{g}$ dek55-1 kernels. Scale bars = $1 \mathrm{~mm}$. $\mathbf{h}$ Hundred-kernel weight of WT and dek55-1 kernels at maturity. (The asterisks indicate significant differences; ${ }^{* *}, P<0.05$, Student's $t$-test.). i-I Histological analysis of WT and dek55-1 kernels at 12 and 18 DAP. $\mathbf{i}$ and $\mathbf{k}$ WT at 12 and 18 DAP, respectively. $\mathbf{j}$ and $\mathbf{I}$ dek55-1 kernels at 12 and 18 DAP, respectively. Scale bars $=1 \mathrm{~mm}$. En, endosperm; Em, embryo; P, pericarp; LP, leaf primordium; RAM, root apical meristem; SAM, shoot apical meristem; SC, scutellum; COL, coleoptile; COR, coleorhiza; C, cavity

(Fig. 1i-1). At 12 DAP, the dek55-1 embryo had only a small scutellum whose development was arrested at the coleoptile stage and a large interspace between the endosperm and the seed coat. In contrast, the WT embryo contained a visible coleoptile, a shoot apical meristem, a scutellum, and two leaf primordia, and the kernel was filled with endosperm cells (Fig. 1i, j). At 18 DAP, the WT embryo had developed complete structures, including four leaf primordia, a shoot apical meristem, and a clearly visible root apical meristem (Fig. 1k), while the dek55-1 embryos presented only a single leaf primordium (Fig. 11). In addition, fewer starch grains accumulated in the dek55-1 endosperm cells than in the WT endosperm cells at this stage (Fig. $1 \mathrm{k}, \mathrm{l}$ ), and a cavity was observed in the dek55-1 endosperm (Fig. 11). Taken together, these results indicate that developmental defects in the embryo and endosperm had occurred in the dek55-1 mutant. 


\section{Map-based cloning of DEK55}

To identify the DEK55 gene, we applied the classic mapbased cloning strategy to identify filial $2\left(\mathrm{~F}_{2}\right)$ mutant kernels, which segregated from a self-pollinated $F_{1}$ hybrid ear. Four genomic DNA pools (10 mutant kernels per pool) and the DNA of both of the parents were used to identify the chromosomal location of the DEK55 gene. Six simple sequence repeat (SSR) markers on chromosome 5 were strongly correlated with defective kernel phenotypes, suggesting that the candidate gene may be on chromosome 5. Further analysis showed that the DEK55 gene is located between umc1705 and umc2302 on chromosome 5 (Fig. 2a). One thousand eight hundred and sixty-eight mutant kernels from the $F_{2}$ population were genotyped to narrow the gene location by the use of six polymorphic molecular markers. The DEK55 gene was ultimately located on an approximately $1.29 \mathrm{Mb}$ region between molecular marker 3 (M3) and M4 (Fig. $2 \mathrm{a})$. There are 25 putative protein-coding genes in this region (http://ensembl.gramene.org/Zea_mays/Info/ Index). To identify the mutated genes, the genomic DNA of 25 candidate genes was amplified and sequenced. Sequence alignment revealed a single- nucleotide polymorphism in the E-subgroup PPR protein

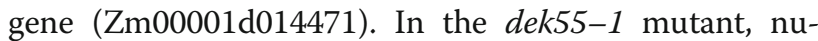
cleotide $\mathrm{C}$ was replaced with nucleotide $\mathrm{T}$ at $+449 \mathrm{bp}$, resulting in the substitution of the amino acid serine (Ser) with phenylalanine (Phe). However, no change in the mRNA expression level was observed (Fig. 2a-d). To validate our results, we obtained a new mutant, dek55-2, from the maize ethylmethanesulfonate-induced mutant database [34]. The dek55-2 mutant showed a singlenucleotide mutation ( $G$ to A) at +729 bp (Fig. 2b), which led to a truncated protein (Fig. 2d). The mutant dek55-2 also produced defective kernels with a small white pericarp (Fig. 2e). An allelic test between dek55-1 and dek55-2 heterozygotes revealed that normal and mutant kernels segregated at the expected 3:1 ratio (normal/mutant; $450 / 143 ; P=0.62$ ) in the $F_{1}$ ear (Fig. 2e). For a control, all the kernels from the ear that were crossed between the dek55-2 heterozygote and WT were normal (Fig. 2e). Taken together, these results indicate that the mutation in the Zm00001d014471 PPR gene was responsible for the defective kernel phenotype, so the annotated gene was designated DEK55.

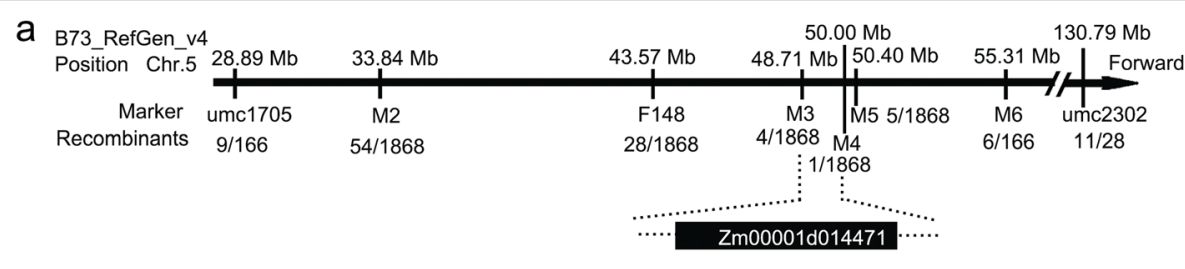

b

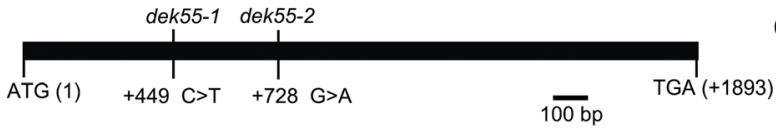

d
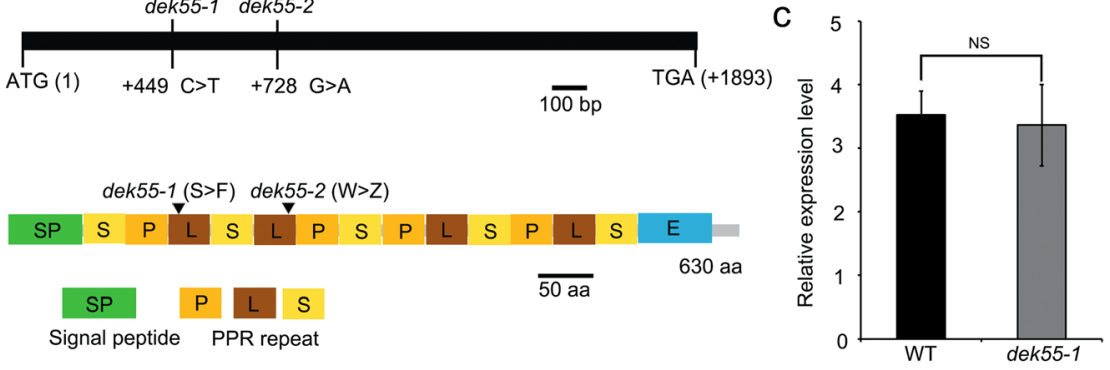

e
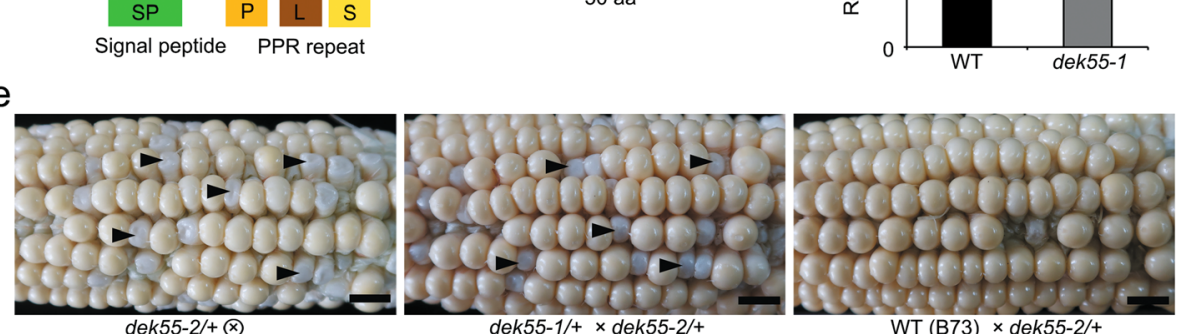

Fig. 2 Map-based cloning and identification of DEK55. a Fine mapping of the DEK55 locus. The DEK55 locus was mapped to a 1.29 Mb region between marker 3 (M3) and M4 on chromosome 5, in which there are 25 candidate genes. The physical location of polymorphic molecular markers and the number of recombinants are shown in the schematic diagram. $\mathbf{b}$ Schematic structure of the dek55 gene. The mutation sites of dek55-1 and dek55-2 are shown. c Relative expression level of DEK55 in WT and dek55-1 kernels. The values are the means of three biological replicates. The error bars represent the standard deviations. (Not significant (NS); $P>0.05$, Student's $t$-test). d Schematic diagram of the DEK55 protein, which contains a total of 13 PPR domains (P, L and S) and an E domain. The amino acid changes in dek55-1 and dek55-2 are indicated. e The self-pollinated dek55-2/+ (heterozygote) at 15 DAP, dek55-1/+ and dek55-2/+ were used in an allelism test of dek55. A cross between dek55$2 /+$ with B73 (WT) was used as a control. Several mutant kernels are indicated by the black arrowheads. Scale bars $=1 \mathrm{~cm}$ 
DEK55 is a mitochondrial E-subgroup PPR protein

Sequence alignment demonstrated that the DEK55 gene is a $1893 \mathrm{bp}$ long ORF with no introns. Moreover, DEK55 encodes a 630 amino acid protein containing 13 PPR motifs and an E domain at the carboxy-terminal end (Fig. 2b-d and Additional file 1: Fig. S1). Mutations in dek55-1 and dek55-2 were located in the third and fifth PPR motifs, respectively (Fig. 2d). The mutation in dek55-2 resulted in a truncated DEK55 protein missing the last eight PPR motifs and the E domain.

To examine the subcellular localization of DEK55, a p35S:DEK55-enhanced green fluorescent protein (EGFP) vector was constructed and transformed into maize protoplasts. The fluorescent signal of DEK55-EGFP overlapped with that of MitoTracker (a mitochondrionspecific dye) (Fig. 3a), suggesting that, in maize, DEK55 is a mitochondrial PPR protein (Fig. 3a). In addition, expression analysis in various maize tissues demonstrated that DEK55 is relatively highly expressed in the roots, anthers, and ears, with relatively low expression in the stems, leaves, silk, tassels, and kernels (Fig. 3b).

\section{DEK55 is involved in the C-to-U editing of 14 transcripts at multiple sites}

PPR proteins usually participate in modifying organelle transcripts [10]. It has been reported that E-subgroup PPRs are involved in the C-to- $\mathrm{U}$ editing of mitochondrial pre-mRNAs $[14,32,33]$. To explore whether DEK55 is involved in this processing, the transcript levels of 35 maize mitochondrial genes that encode functional proteins were analysed in both WT and dek55-1 kernels. RNA editing of these transcripts in the dek55 (dek55-1 and dek55-2) and WT (WT-1 and WT-2) kernels was detected via the strand- and transcript-specific RNA sequencing (STS-PCRseq) strategy [35]. The sequencing reads were mapped to the 35 mitochondrial gene transcripts, and $482 \mathrm{C}$-to-U RNA editing sites were examined in the WT and dek55 kernels (Additional file 2: Table S1). The results revealed that, compared with that of these RNA editing sites between the WT and dek55 kernels (Additional file 2: Table S2), the C-to-U editing percentage of 31 editing sites in 14 transcripts (atp1, atp8, ccmFc, ccmFn, cob, mat-r, nad3, nad4, nad6, nad7, rps12-ct, rps12, rps13, and rps3) was significantly altered in the dek55 kernels (Fig. 4, Additional file 2: Tables S2S4), whereas the editing percentage of 24 sites decreased (Fig. 4a) and that of seven sites increased in the dek55 kernels compared with WT kernels (Fig. 4b, Additional file 2: Tables S2 and S4). The editing efficiency at the atp1-1490, ccmFn-287, mat-r-1877, and rps13-56 sites dramatically decreased in the dek55-1 and dek55-2 kernels, and the editing percentage in the dek55 mutants was more than $50 \%$ lower than that in the WT kernels (Fig. 4a, Additional file 2: Table S3). Direct sequencing of reverse transcription-polymerase chain reaction (RTPCR) products of the atp1-1490, ccmFn-287, mat-r1877 and rps13-56 sites also indicated that the editing efficiency was significantly reduced in the dek55 kernels at these RNA editing sites (Fig. 4c). Deficient C-to-U RNA editing led to altered amino acid residues in dek55 (Fig. 4c). Moreover, at the atp8-123 site, the editing efficiency of only the dek55-2 kernels (5\%) was more than $50 \%$ lower than that of the WT kernels, and at nad4-77 sites, the editing efficiency of only the dek55-1 kernels (24.2\%) was more than $50 \%$ lower than that of the WT kernels (Fig. 4a). Taken together, the above results indicated that DEK55 is required for RNA editing at multiple sites, especially the atp1-1490, ccmFn-287, mat-r1877, and rps13-56 sites.

DEK55 is essential for the trans-splicing of nad1 introns 1 and 4 and the cis-splicing of nad4 intron 1

The transcript levels of 35 maize mitochondrial genes were examined, and the results showed that the transcript levels of nad1 and nad4 were significantly downregulated in the dek55 mutants (Fig. 5a). The genomic DNA of nad1 contains four group II introns, and with the exception of the 2nd intron, all are trans-splicing introns. (Fig. 5c). The genomic DNA of nad4 has three cis-splicing introns (Fig. 5d) [13, 36]. The full maturation of nad1 and nad4 transcripts requires complete intron splicing. We therefore further analysed the intron splicing efficiency of nad1, nad4, and other genes in the dek55-1 and WT kernels via quantitative reverse transcription-polymerase chain reaction (qRT-PCR). Compared with that in the WT kernels, the splicing efficiency of the first and fourth introns of nad1 and the first intron of nad4 in the dek55-1 mutant kernels decreased (Fig. 5b). Furthermore, we amplified each intron and full transcript of nad1 and nad4 via RT-PCR (Fig. $5 \mathrm{c}, \mathrm{d})$. The transcript abundance of nad1 exons 1-2 and exons 4-5 and the full-length DNA fragments significantly decreased (Fig. 5c). RT-PCR could not amplify the intronic DNA fragments $(1 \mathrm{~F}+2 \mathrm{R}, 3 \mathrm{~F}+4 \mathrm{R}, 4 \mathrm{~F}+5 \mathrm{R})$ in the dek55 and WT kernels because the 1st, 3rd, and 4th introns of nad1 are trans-spliced. (Fig. 5c). The unspliced 2nd intronic fragments of nad1 in the dek55 mutant kernels were similar to those in the WT kernels (Fig. 5c). The abundance of nad4 spliced exons 1-2 and full-length DNA fragments significantly decreased, and the abundance of nad4 unspliced intron 1 transcripts significantly increased (Fig. 5d). Our findings suggest that the significant decrease in abundance of nad4 and nad1 transcript in the dek55 mutant kernels was caused by the abnormal splicing of nad4 intron 1, nad1 intron 1 , and intron 4 (Fig. 5a-d). Therefore, DEK55 is necessary for the trans-splicing of the two nad1 introns (1st 

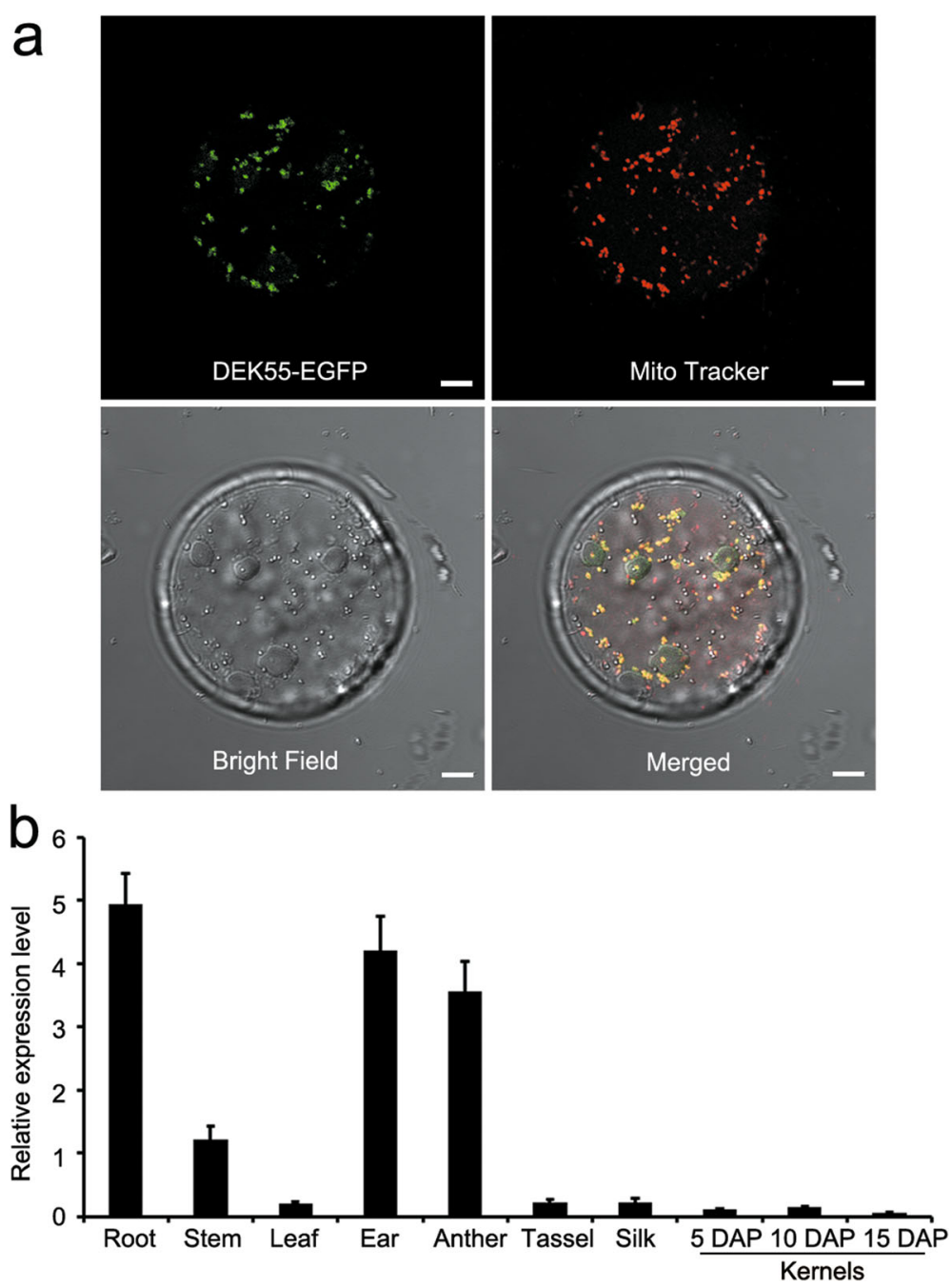

Fig. 3 Subcellular localization of DEK55 and expression pattern of DEK55. a The subcellular localization of DEK55 was determined by transient expression of DEK55-EGFP fusion proteins in maize protoplasts. The mitochondria are stained by MitoTracker (red). Scale bars $=5 \mu \mathrm{m}$. $\mathbf{b}$ Analysis of the relative expression level of DEK55 in various tissues and kernels at 5, 10, and 15 DAP. The ZmActin gene (GRMZM2G126010) was used as an internal control. The values are the means of three replicates. The error bars represent the standard deviations

and 4th) and cis-splicing of the first nad4 intron in maize.

The dek55-1 mutant exhibits reduced complex I activity and increased alternative respiratory pathway activity Four genes, i.e., nad1, nad4, nad3, and nad6, encode the subunits of complex I NAD1, NAD4, NAD3, and NAD6, respectively [36]. The rps13 gene encodes a ribosomal protein, and atp 1 and atp 8 encode the ATPase subunit 1 and subunit 8 subunits of ATP synthase F1, respectively [36]. Defects in the posttranscriptional processing of these genes may impair the biosynthesis of mitochondrial complexes [17, 37-39]. We performed blue native polyacrylamide gel electrophoresis (BN-PAGE) and an in-gel NADH dehydrogenase activity assay to investigate the accumulation level and activity of mitochondrial complexes in WT and dek55-1 endosperm. BN-PAGE showed that the abundance of complex I and supercomplex $\mathrm{I}+\mathrm{III}_{2}$ in the dek55-1 mutant significantly decreased (Fig. 6a). However, no significant differences for complex $\mathrm{V}$ were observed between the WT endosperm and dek55-1 endosperm (Fig. 6a). Furthermore, the activity of complexes $\mathrm{I}$ and $\mathrm{I}+\mathrm{III}_{2}$ was reduced in the dek55-1 mutant (Fig. 6b). Taken together, these results indicate that defects in mitochondrial transcript splicing and/or editing might affect the abundance and activity of mitochondrial complex I.

The mitochondrial respiratory chain in plants includes the cytochrome $c$ and alternative oxidase (AOX) pathways [40]. When the main cytochrome $c$ pathway is blocked, AOX activity can be increased to compensate for the respiratory pathway [41]. In $\operatorname{dek} 55-1$, the 


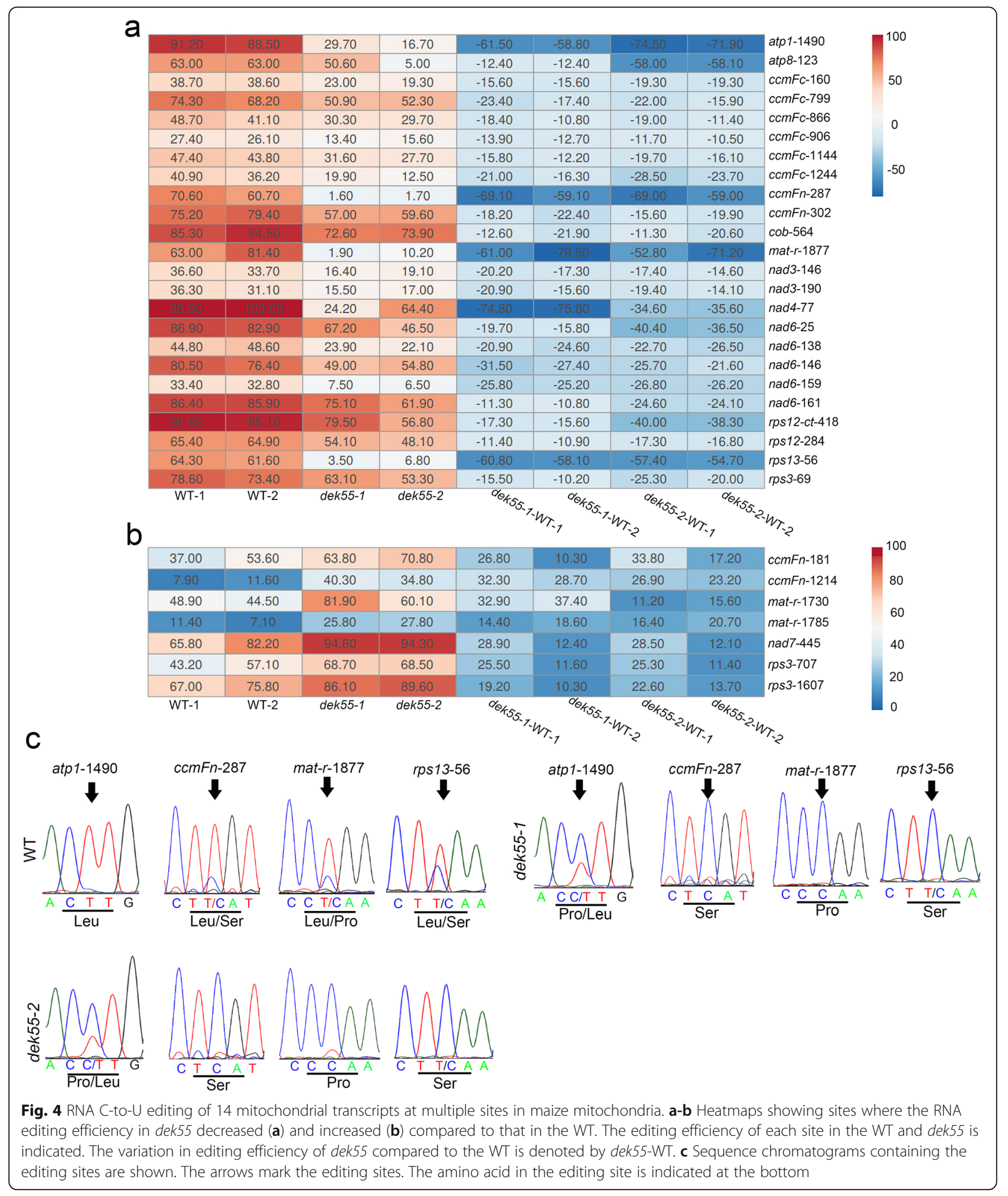




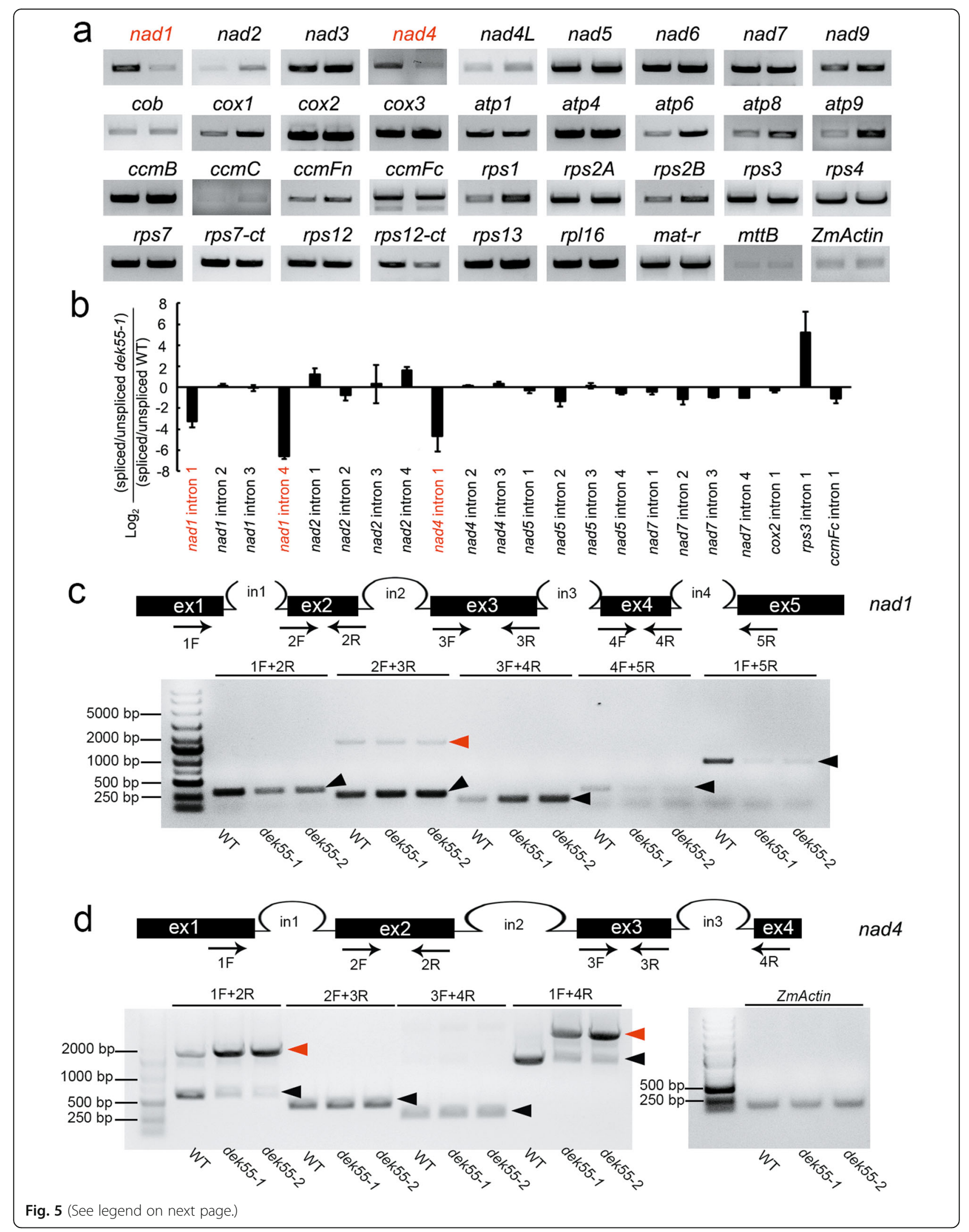


(See figure on previous page.)

Fig. 5 The posttranscriptional RNA processing of nad 1 and nad4 was affected in dek55. a The expression of 35 mitochondrion-encoded genes in the WT (left) and dek55-1 (right) kernels was detected via RT-PCR. The ZmActin gene (GRMZM2G126010) was used as an internal control. Both nad1 and nad4 are marked in red because their transcript abundance significantly decreased. $\mathbf{b}$ The splicing efficiency of all 22 group II introns in maize mitochondrial-encoded genes was determined in dek55-1 and WT kernels via qRT-PCR. The values shown are the means of three biological replicates, and the error bars represent the standard deviations. c-d Schematic structure of the nad1 gene (c) and nad4 gene (d). The primers used for amplification are indicated. RT-PCR-based analysis of the intron splicing efficiency of nad1 in WT kernels and in dek55-1 and dek55-2 mutant kernels at 15 DAP. All the PCR products were confirmed by sequencing. The ZmActin gene (GRMZM2G126010) was used as an internal control. The unspliced and spliced fragments are indicated by red and black arrowheads, respectively. Exons are indicated as "ex", and introns are indicated as "in". The gel images in $(\mathbf{a}, \mathbf{c}, \mathbf{d})$ were cropped; the original gel images are shown in Additional file 1: Figs. S2-S3

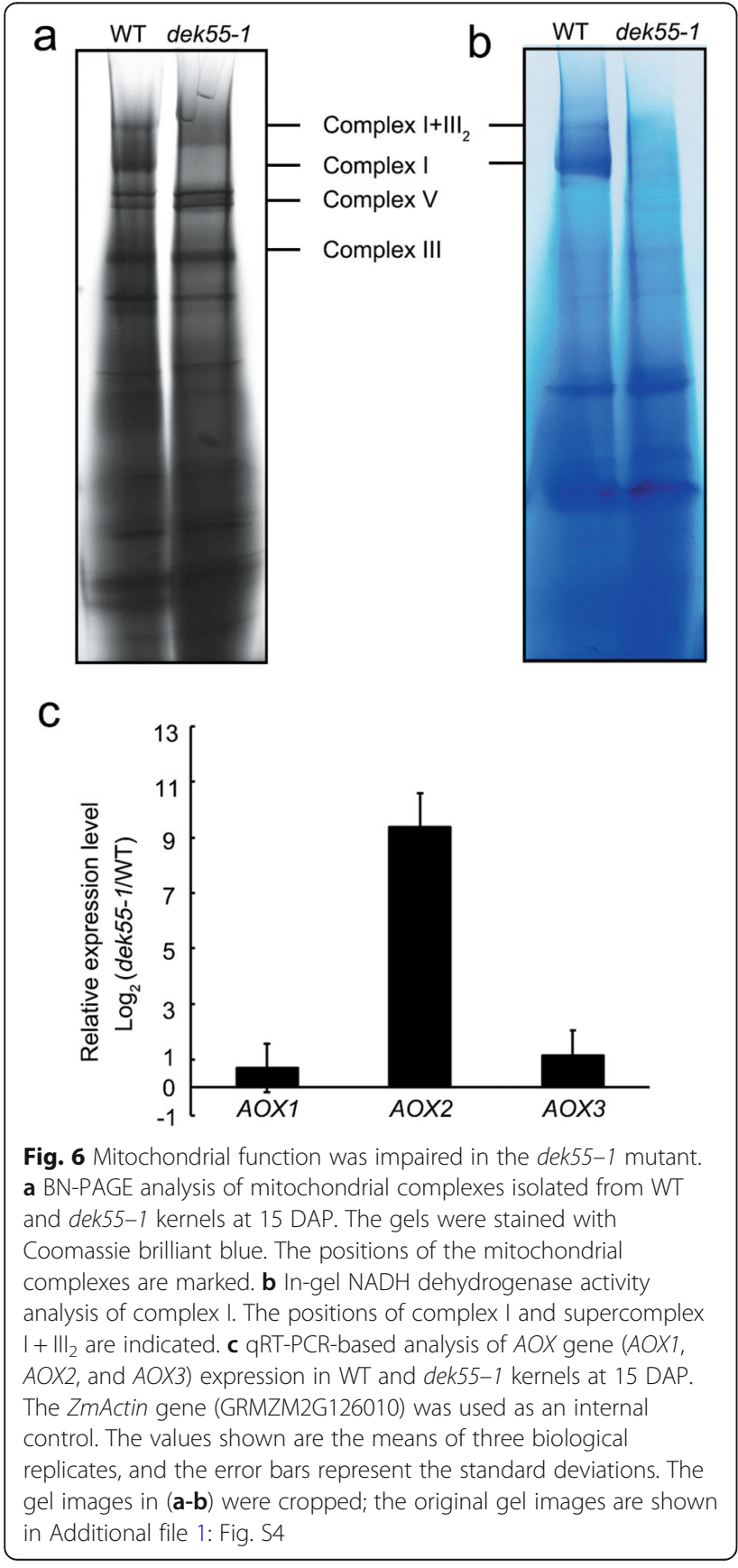

functions of complex I were abolished (Fig. 6a, b). Thus, we performed qRT-PCR to detect the expression levels of $A O X$ genes in WT and dek55-1 kernels, and the results showed a 512 -fold increase in the expression level of the AOX2 gene in the dek55-1 mutant kernels compared to the WT kernels. (Fig. 6c). Collectively, our results indicate that the respiratory pathway is severely blocked in dek55-1 mitochondria.

\section{DEK55 interacts with ZmMORF1 and ZmMORF8 in yeast}

Previous studies have shown that MORFs directly interact with PPR proteins and play a role in RNA editing at numerous editing sites $[42,43]$. In the present study, DEK55 was found to be responsible for 31 RNA editing events in maize, so we speculated that DEK55 might interact with MORFs to form an editing complex involved in RNA editing in maize. Thus, we used MORFs in Arabidopsis as bait to search for putative MORFs in maize; seven putative MORFs were identified in maize (Fig. 7a). A yeast two-hybrid assay was performed to screen for MORFs that interact with DEK55, and the results indicated that DEK55 can interact with ZmMORF1 and ZmMORF8 in yeast (Fig. 7b).

\section{Discussion}

\section{DEK55 is required for maize kernel development}

Previous reports have shown that PPR proteins play vital roles in maize kernel development, and the loss of function of some PPR proteins results in empty pericarp and small and defective kernel phenotypes in different genetic backgrounds [13, 14, 17-21, 30, 31, 39, 44, 45]. The ppr mutants exhibit developmentally arrested embryos and endosperm. The embryos usually reached the coleoptile stage or leaf stage 1 (L1), and the endosperm presented significantly reduced starch and protein levels $[14,33,45]$. The dek55 mutants produced small kernels with a shrivelled pericarp (Fig. 1a-c). Moreover, compared with the WT kernels, the mutant kernels had smaller embryos and a reduced proportion of hard endosperm (Fig. 1d-1). In particular, dek55-1 embryos were severely arrested and had only one leaf primordium. Thus, these mutant kernels would not be able to germinate in the field. Allelic tests indicated that the nonsense mutant dek55-2, an allelic mutant with dek55-1 and dek55-1/dek55-2 heterozygous kernels, exhibited a phenotype similar to that of $\operatorname{dek55-1.}$ 


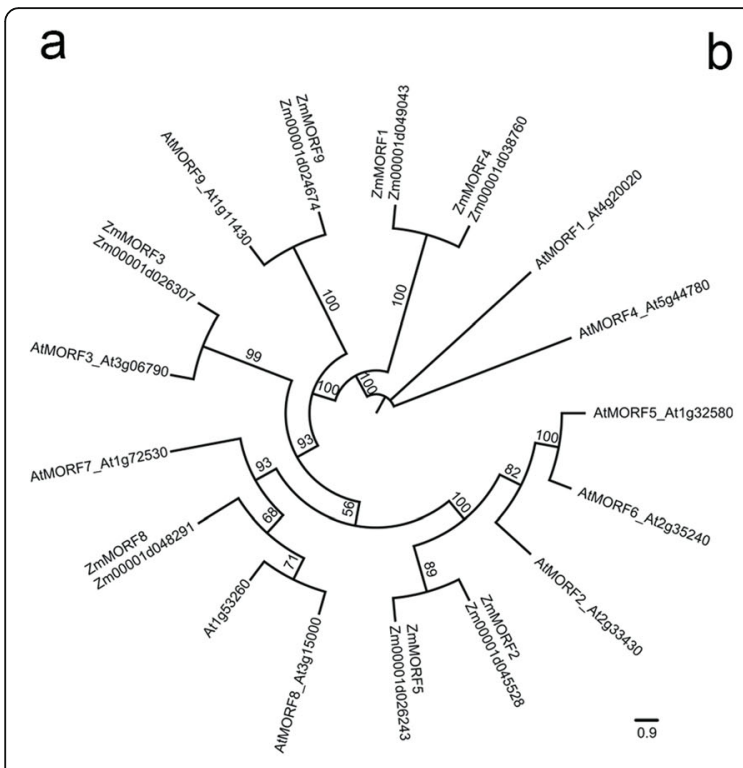

Fig. 7 DEK55 interacts with ZmMORF1 and ZmMORF8 in yeast. a Phylogenetic tree of known AtMORFs in Arabidopsis and putative ZmMORFs in maize. $\mathbf{b}$ Yeast two-hybrid assay of the interaction between DEK55 and ZmMORFs. Yeast cells diluted to different concentrations $\left(1,5^{-1}, 5^{-2}, 5^{-3}\right.$, $5^{-4}$ ) were cultured in SD/-Trp-Leu dropout and SD/-Trp-Leu-His-Ade plates supplemented with X-a-gal dropout media at $30^{\circ} \mathrm{C}$, and the results were recorded after 3 days of culture. The images in (b) were cropped; the original gel images are shown in Additional file 1: Fig. S5
This suggests that dek55 dysfunction is responsible for the defective kernel phenotype and that DEK55 is required for kernel development in maize.

The E-subgroup PPR proteins are characterized as having an E domain at their carboxy-terminal end, and this domain might be responsible for interactions between proteins $[46,47]$. In the dek55-1 mutant, there was a single-nucleotide change ( $\mathrm{C}$ to $\mathrm{T})$ at +449 in the dek55 gene, which resulted in Phe instead of Ser on the third PPR motif of DEK55 at position 150 in the polypeptide chain (Fig. 2b, d; Additional file 1: Fig. S1). This mutation from a hydrophilic amino acids to a hydrophobic amino acid would alter the affinity of the protein to water. Our evidence suggests that this amino acid change (Ser $\rightarrow$ Phe) is responsible for defective kernels in the dek55 mutants. Therefore, the amino acid change at this site in DEK55 might cause conformational changes and loss of function. In the dek55-2 mutant, the mutation resulted in a loss of the last eight PPR motifs and E domain at the carboxyterminal end of the DEK55 protein (Fig. 2b, d), which might prevent it from forming complexes with other proteins and binding to its target sites.

DEK55 is necessary for the C-to-U editing of multiple sites in the mitochondrial transcripts of maize

PPR proteins, including DYW2, EMP21, NUWA, MEF8, and DEK53, are involved in C-to-U editing at multiple sites [48-52]. In this study, we demonstrated that DEK55 is involved in RNA editing at 31 sites; however, the editing percentage of 24 editing sites and that of seven editing sites decreased and increased, respectively, in dek55, suggesting that DEK55 is also necessary for RNA editing at multiple sites. Among them, DYW2 and MEF8 harbour only five PPR repeats and belong to an atypical DYW subgroup [48, 49]. NUWA belongs to the P-class of PPR proteins [48, 50], and EMP21 contains 11 PPR motifs in addition to the E and DYW domains and belongs to the PPR-DYW protein family [51]. Furthermore, DEK53 is an E-subgroup PPR protein with seven PPR repeats [52], and DEK55 is considered an E-subgroup PPR protein that contains the canonical E domain. Therefore, PPR proteins that target multiple sites for editing might have dissimilar structures.

MORFs can interact directly with PPR proteins to participate in RNA editing [42, 43, 51]. In Arabidopsis, MEF13 (an E-subgroup PPR protein) interacts with MORF3 and MORF8; this protein complex is responsible for RNA editing of the same sites among morf3, morf8, and mef13 mutants [42]. EMP21 is necessary for the editing of $\sim 17 \%$ of mitochondrial target $\mathrm{Cs}$ in maize [51]. Interestingly, 34 editing sites overlap in maize emp 21 mutants and Arabidopsis morf8 mutants, and eight editing sites overlap in maize emp5 mutants and morf8 mutants. ZmMORF8 (GRMZM2G169384), an orthologue of MORF8 in maize, directly interacts with EMP21 and EMP5, suggesting that EMP21 and EMP5 participate in the editing of several sites by interacting with ZmMORF8 [51]. DEK53 is an E-subgroup PPR protein that interacts with ZmMORF1 to form an RNA-editing complex and is responsible for more than 60 RNA edits in the maize 
mitochondrion [52]. In our study, DEK55 participated in the C-to-U editing of 14 mitochondrial transcripts at 31 editing sites, while the editing percentage of 24 sites decreased in dek55 (Fig. 4a, Additional file 2: Table S3) (Fig. 4). Moreover, comparative analyses of these mitochondrial transcript C-to$\mathrm{U}$ editing events in both Arabidopsis and maize indicated that multiple sites, e.g., $c c m F c-799, c c m F c-866, c c m F c-1144$, ccmFc-1244, cob-564, mat-r-1877, nad3-190, nad4-77, and nad6-146, were not edited in Arabidopsis, as these sites are "Ts". This suggests that editing of these sites is necessary in maize. In addition, editing was also substantially impaired in Arabidopsis morf8 mutants at the following four sites: atp11484 (atp1-1490 in the dek55 mutants), ccmFc-160, nad6161, and rps12-284 [35]. DEK55 can interact with ZmMORF8 (orthologue of AtMORF8 in maize), as shown by yeast two-hybrid assays. Although a Zmmorf8 mutant has not yet been identified in maize, our findings might provide evidence that DEK55 might interact with ZmMORF8 to function at these RNA editing sites in maize. In dek53, more than 60 RNA editing sites were affected [52], and three of them (nad3-146, nad6-146, and atp8-123) were also affected in dek55. DEK53 and DEK55 interact with ZmMORF1 [52, this study]. Therefore, DEK53 and DEK55 might be responsible for C-to-U RNA editing of these sites by interacting with ZmMORF1. Taken together, these results indicate that ZmMORF8 and ZmMORF1 might interact with DEK55 to form an editing complex for these multiple editing sites.

\section{DEK55 is involved in group II intron splicing in maize mitochondria}

E-subgroup PPR proteins are considered editing factors for RNA editing in organelles [10], but few of these proteins are considered to play a role in splicing $[28,29,53]$. SLO4 is necessary for RNA editing of nad4 and the efficient splicing of nad2 intron 1 in Arabidopsis mitochondria [29]. AEF1/MPR25 is involved in RNA editing of atpF and nad5 and modulates atpF splicing in both Arabidopsis and rice [28]. Furthermore, the plastid PPR protein OTP70 participates only in the intron splicing of the rpoC1 transcript [53]. In this study, DEK55 (an Esubgroup PPR protein) was shown to participate in both the RNA editing of 31 sites and group II intron splicing in maize mitochondrial transcripts (Figs. 4 and 5a-d). The RNA editing percentage of nad1 transcripts was not affected, and among the nad4 transcripts, only nad4-77 transcripts decreased. Moreover, it has been reported that intron splicing can be mediated by RNA editing events in which the key sites of introns are edited [54-56]. The editing efficiency of two RNA editing sites (147 and 409) on nad4 intron 1 increased in dek55 (Additional file 2: Table S1), which might have resulted from the reduction in the splicing efficiency of nad4 intron 1. Since splicing of nad1 introns 1 and 4 occurs in trans, the introns were unable to be amplified via $\mathrm{RT}$-PCR with primer pairs $(1 \mathrm{~F}+2 \mathrm{R}$ and $4 \mathrm{~F}+5 \mathrm{R}$ ) (Fig. 5c). The editing efficiency of the RNA editing sites on nad 1 introns 1 and 4 were not analysed. Thus, it could not be confirmed whether the decreased splicing efficiency of nad 1 introns 1 and 4 in $\operatorname{dek} 55$ was caused by editing events of these introns.

Several proteins that participate in the splicing of nad1 and nad4 pre-mRNAs have been identified. Nuclear maturase 1 [57], DEK2 [45], and EMP11 [44] participate in the trans-splicing of nad1 intron 1, and EMP11, EMP8, and ZmSMK3 are required for nad1 intron 4 trans-splicing [44]. The proteins NMS1 [58], DEK35 [19], EMP8 [13], DEK43 [20], EMP602 [59], and ZmSMK3 [60] and are involved in cis-splicing of nad4 intron 1 . The results of our study demonstrated that DEK55 is involved in both trans- and cis-splicing. It appears that splicing of one intron may require the involvement of multiple factors to constitute a putative spliceosome. This is supported by the finding that PPR-small MutS-related-1 can interact with Zm-mCSF1 to form a protein complex. This protein complex is subsequently involved in the intron splicing of multiple transcripts within the mitochondria [61]. Therefore, DEK55 might interact with P-type PPR proteins or other splicing factors involved in group II intron splicing.

\section{Conclusions}

In this study, we demonstrated that DEK55 is a mitochondrion-localized E-subgroup PPR protein. Mutation of DEK55 leads to embryo lethality and arrested endosperm development in maize. DEK55 is required for editing at 31 RNA editing sites, especially the atp11490, ccmFn-287, mat-r-1877, and rps13-56 sites (Fig. 4). Moreover, DEK55 can interact with ZmMORF1 and ZmMORF8 in yeast. In addition, DEK55 is responsible for the trans-splicing of two nad1 introns (intron 1 and intron 4) and the cis-splicing of nad4 intron 1 in the mitochondria. Our results suggest that the E-subgroup PPR protein DEK55 plays important roles in the RNA editing and splicing of introns of maize mitochondrial transcripts. These results provide a novel perspective for understanding the molecular function of E-subgroup PPR proteins in RNA processing in plant organelles.

\section{Methods \\ Plant materials}

The maize mutant dek55-1 identified from an ethylmethanesulfonate population in the B73 background was kindly provided by Prof. Xiaoduo Lu of Qilu Normal University. The original name of the allele mutant dek55-2 was EMS4-073342, which was purchased from a maize ethylmethanesulfonate-induced mutant database (http://www.elabcaas.cn/memd/) [34] and identified by searching for the gene ID (Zm00001d014471). To purify the genetic background, dek55-1 was back-crossed with 
the $\mathrm{B} 73$ inbred line, and $\mathrm{BC}_{2} \mathrm{~F}_{2}$ kernels were used in this study. A dek55-1 heterozygote (as the male parent) was crossed with our laboratory-grown inbred lines C733 and S162, after which the $F_{1}$ progeny were selfpollinated to generate an $F_{2}$ population that was used for gene mapping. Ru Chang Ren and Xu Wei Yan formally identified the plant materials. All the plant materials were sown at the experimental station of Shandong Agricultural University (Taian, Shandong Province).

\section{Histological analysis}

WT and defective kernels were obtained from selfpollinated heterozygous plants at 12 and 18 DAP, respectively. The middle part of the kernel along the longitudinal axis was selected and placed in formalin-acetic acid-alcohol solution for at least $12 \mathrm{~h}$ on ice, followed by treatment with 50, 70, 85, 95, and 100\% ethanol. Afterwards, the seeds were placed in $100 \%$ xylene for $2-4 \mathrm{~h}$. After dehydration, the materials were immersed in molten paraffin for $72 \mathrm{~h}$ at $60^{\circ} \mathrm{C}$ and then embedded in the paraffin. The paraffin-embedded samples were cut into $12 \mu \mathrm{m}$ slices using a microtome (Leica RM2235, Germany). The sections were then stained based on the methods of Ren et al. [20]. The sections were ultimately imaged with a light microscope equipped with a camera (Olympus DP72, Olympus, Tokyo, Japan).

\section{Map-based cloning}

The DEK55 locus was identified using $1868 \mathrm{~F}_{2}$ defective kernels from the self-pollinated $F_{1}$ population $(\mathrm{C} 733 \times$ dek55-1/+). For preliminary mapping, 73 polymorphic SSR markers selected from the entire genome were used to screen the DNA of the parents, individual $F_{1}$ plants, and four groups of pooled $F_{2}$ defective kernels. For fine mapping, new molecular markers were selected according to the parental DNA sequences. A website (http:// ensembl.gramene.org/Zea_mays/Info/Index) was used to search for the genes annotated in the candidate regions of the Zea mays genome (B73_RefGen_v4) [62]. Phanta EVO Super-Fidelity DNA polymerase (catalogue number P503d1, Vazyme Biotech Co., Nanjing, China) was used to clone all the candidate gene genomic DNA sequences and sequencing. The primers used were designed according to the candidate gene reference sequences. The primers used to clone the full-length $D E K 55$ gene and used for mapbased cloning are given in Additional file 1: Table S2.

\section{RNA extraction, RT-PCR, and qRT-PCR}

Total RNA from WT and dek55 mutant kernels without a pericarp and other tissues was extracted with an Ultrapure RNA Kit (CWBIO, China). Any residual DNA among the total RNA was removed by DNase. For RTPCR, complementary DNA (cDNA) was obtained by reverse transcription and used as the template for polymerase chain reaction (PCR)-based amplification. KOD DNA polymerase (KOD FX Neo, code: KFX-201, Toyobo, Japan) was used for PCR. The PCR procedure was as follows: initial melting at $94{ }^{\circ} \mathrm{C}$ for $2 \mathrm{~min}$; 33 to 38 cycles of $15 \mathrm{~s}$ at $98^{\circ} \mathrm{C}$ and $30 \mathrm{~s}$ at the applicable annealing temperature $\left(58^{\circ} \mathrm{C}\right.$ to $\left.61^{\circ} \mathrm{C}\right)$ for the various primer pairs; adequate extension $(30 \mathrm{~s} / \mathrm{kb})$ at $68^{\circ} \mathrm{C}$; and a final extension at $68^{\circ} \mathrm{C}$ for $7 \mathrm{~min}$. RT-PCR was performed to amplify the mitochondrial transcripts, splicing efficiency of nad1 and nad4 introns. The DNA fragments obtained via RT-PCR were directly sequenced. The transcripts were amplified by the use of previously reported primers [63], which are listed in Additional file 1: Table S2. The primers used to amplify the introns of nad1 and nad4 are shown in Additional file 1: Table S2.

The qRT-PCR equipment and reaction system were the same as those of a previous report [20]. All the qRTPCR assays were performed for three samples and with three technical repeats. The primers for group II intron splicing efficiency analysis in the mitochondria were designed according to previous reports [17, 18, 63]. The primers used to analyse DEK55 expression levels are shown in Additional file 1: Table S2.

\section{RNA editing efficiency analysis}

The RNA editing of 35 mitochondrial genes was analysed via the STS-PCRseq method described by Bentolila et al. [35], with slight modifications. The 35 mitochondrial gene transcripts and nad4 intron 1 were amplified through RT-PCR using four cDNA libraries as templates. These cDNA libraries were obtained from WT and dek55 mutant kernels (WT-1 and dek55-1; WT-2 and dek55-2) obtained from self-pollinated dek55-1/+ and dek55-2/+ heterozygous ears at 15 DAP. The PCR products were visualized on $1 \%$ agarose gels; the gel bands were excised, and the DNA fragments were purified using a Gel DNA Extraction Mini Kit (catalogue number DC301-01, Vazyme Biotech Co.,). Purified DNA samples amplified from the same cDNA library were mixed together in equimolar amounts and sonicated to generate $300-500 \mathrm{bp}$ DNA fragments. The DNA library was constructed and sequenced on the Illumina platform by Novogene Bioinformatics Technology Co., Ltd. (Beijing, China). The sequenced data were filtered to remove any low-quality reads, adaptor reads, and unknown base reads. The clean reads were subsequently mapped to the 35 mitochondrial gene transcripts and nad4 intron 1 using Bowtie 2, and allele frequency counts were performed as described previously [52]. The editing efficiencies of 482 RNA editing sites were calculated, and the affected editing site in the dek55 mutants was defined as described previously [51]. Editing efficiency was considered to have decreased in the dek55 mutants when the $\mathrm{T} /(\mathrm{T}+\mathrm{C}) \%$ in $\operatorname{dek} 55-\mathrm{T} /(\mathrm{T}+\mathrm{C}) \%$ in 
the WT was $\leq-10 \%$, while the editing efficiency was considered to have increased in the dek55 mutants when the $\mathrm{T} /(\mathrm{T}+\mathrm{C}) \%$ in $\operatorname{dek} 55-\mathrm{T} /(\mathrm{T}+\mathrm{C}) \%$ in the WT was $\geq 10 \%$. Overlapping sites affected in dek55-1 vs. WT-1, dek55-1 vs. WT-2, dek55-2 vs. WT-1, and dek55-2 vs. WT-2 were considered affected editing sites [51].

\section{Yeast two-hybrid assays}

The full-length ORF of DEK55, excluding the signal peptide coding sequence (1-207 bp), was amplified using specific primers. The PCR products were subsequently cloned into a pGBKT7 vector (Clontech, Kyoto, Japan) at the EcoRI and BamHI sites to generate a DEK55-BD bait vector. The coding sequences of seven ZmMORFs were then amplified using specific primers. The PCR products were subsequently cloned into the pGADT7 vectors (Clontech) to generate recombinant $\mathrm{ZmMORF-}$ AD prey vectors. The DEK55-BD and ZmMORF-AD recombinant vectors were cotransfected into Y2HGold competent cell (catalogue number YC1002, Weidi Biotechnology Co., Shanghai, China). Empty pGBKT7 and pGADT7 vectors were used as negative controls. The transformed cells were incubated on synthetic dextrose (SD)/-leucine (Leu)-Trp dropout plates and SD/-LeuTrp-His-Ade dropout plates supplemented with $\mathrm{X}$ - $\alpha$-gal at $30^{\circ} \mathrm{C}$ for 3 days. The primers used are listed in Additional file 1: Table S2.

\section{Subcellular localization}

The complete ORF (excluding the stop codon) of the $D E K 55$ gene was incorporated into a pM999-EGFP vector, generating a DEK55-EGFP recombinant vector driven by the CaMV 35S promoter. Subcellular localization was performed as reported previously [64]. In brief, maize mesophyll cell protoplasts were obtained from etiolated leaves by enzymatic hydrolysis as described previously [21]. Recombinant vectors $(20 \mu \mathrm{L}, 15-20 \mu \mathrm{g})$ were added to a $200 \mu \mathrm{L}$ maize protoplast solution, and $220 \mu \mathrm{L}$ of $40 \%$ (w/v) PEG4000 solution was then added and mixed completely, after which the samples were incubated at $23^{\circ} \mathrm{C}$ for 10 $15 \mathrm{~min}$. Afterwards, the protoplasts were washed using a W5 or WI solution and cultured for $12-16 \mathrm{~h}$ in the dark at $23^{\circ} \mathrm{C}$. Before being imaged, the protoplasts were stained with a mitochondrion-specific dye (MitoTracker Red CMXRos, Thermo Fisher Scientific, Waltham, MA, USA), and the samples were observed using a laser confocal microscope (LSM 880, Zeiss, Jena, Germany).

\section{Isolation and analysis of mitochondrial complexes}

A plant mitochondrial isolation kit (catalogue number P0045, Biohao, Wuhan, China) was used to isolate crude mitochondria from WT and dek55-1 seed tissue, excluding the pericarp (at 15 DAP), for BN-PAGE and complex I activity analysis. The collected mitochondrial precipitate was redissolved in $35 \mu \mathrm{L}$ of solution buffer ( $50 \mathrm{mmol} / \mathrm{L}$ bis-Tris, $6 \mathrm{~N} \mathrm{HCl}, 50 \mathrm{mmol} / \mathrm{L} \mathrm{NaCl}, 10 \% \mathrm{w} /$ v glycerol, $0.001 \%$ Ponceau S; pH 7.2) containing $20 \% \mathrm{n}$ dodecyl-b-D-maltoside (Sigma-Aldrich, St. Louis, MO, USA) to a final concentration of $1 \%$ ) and then kept on ice for $30 \mathrm{~min}$. The suspension was then centrifuged at $4{ }^{\circ} \mathrm{C}$, after which the supernatant was collected and loaded on preprepared gradient gels (BN1002BOX, Thermo Fisher Scientific), and electrophoresis was performed according to the manufacturer's instructions. Afterwards, the gels were placed in $100 \mathrm{~mL}$ of fixing solution (methanol: $\mathrm{ddH}_{2} \mathrm{O}$ :acetic acid, 4:5:1) for $30 \mathrm{~min}$ and then transferred to $0.02 \%$ Coomassie R-250 stain (Sigma-Aldrich, St. Louis, MO, USA) for mitochondrial complex abundance analysis. The gel strips were incubated in assay buffer $(25 \mathrm{mg}$ nitro blue tetrazolium and $100 \mu \mathrm{L}$ of NADH $(10 \mathrm{mg} / \mathrm{mL})$ combined with $10 \mathrm{~mL}$ of $5 \mathrm{mmol} / \mathrm{L}$ Tris/HCl; $\mathrm{pH} 7.4$ ) (Sigma-Aldrich) for $5 \mathrm{~min}$, and the reaction was terminated with the fixing solution $(40 \%$ methanol:10\% acetic acid $(\mathrm{v} / \mathrm{v}))$ for analysis of complex I activity [44].

\section{Supplementary Information}

The online version contains supplementary material available at https://doi. org/10.1186/s12870-020-02765-X.

Additional file 1: Fig. S1. Amino acid alignment of maize DEK55 with homologous PPR proteins of other plant species. Fig. S2. Original gel images corresponding to Fig. 5a. Fig. S3. Original gel images corresponding Fig. 5c-d. Fig. S4. Original gel images corresponding to Fig. 6a-b. Fig. S5. Original gel images corresponding to Fig. 7b. Table S1. Genetic analysis of the mutant kernels in the segregating ear. Table S2. Primers used in this study.

Additional file 2: Table S1. Number of reads at each editing site for each library. Table S2. Editing percentage at each editing site of each library. Table S3. The editing percentage of 24 RNA editing sites decreased in the dek55 mutant kernels. Table S4. The editing percentage of seven RNA editing sites increased in the dek55 mutant kernels.

\section{Abbreviations}

AOX: Alternative oxidase; atp1: ATP synthase subunit 1; BN-PAGE: Blue native polyacrylamide gel electrophoresis; C: Cytidine; cox: Cytochrome c oxidase; CDNA: Complementary DNA; DAP: Days after pollination; dek: Defective kernel; EGFP: Enhanced green fluorescent protein; EMP: Empty pericarp; Leu: Leucine; MEF: Mitochondrial editing factor; MORF: Multiple organellar RNA-editing factor; MPR25: Mitochondrial PPR 25; ORF: Open reading frame; OTP87: Organelle transcript processing 87; PCR: Polymerase chain reaction; Phe: Phenylalanine; PPR: Pentatricopeptide repeat; Pro: Proline; qRTPCR: Quantitative reverse transcription-polymerase chain reaction; rps13: Ribosomal protein S13; RT-PCR: Reverse transcription-polymerase chain reaction; SD: Synthetic dextrose; Ser: Serine; SLO1: Slow growth 1; SMK: Small kernel; SSR: Simple sequence repeat; STS-PCRseq: Strand- and transcriptspecific RNA sequencing; U: Uridine; WT: Wild type

\section{Acknowledgements}

Not applicable.

\section{Authors' contributions}

The experiments were conceived and supervised by XYZ. RCR, YXW, YJZ, YMW, JZ, JWW, and GMZ performed the experiments. XL isolated the dek55- 
1 mutant. The manuscript was drafted by XYZ and RCR and revised by XHD and XSZ. All the authors have read and approved the final manuscript.

\section{Funding}

This work was funded by the National Natural Science Foundation of China (91735301), National Plant Transgenic Program (2016ZX08003-003), Taishan Scholars Project (ts201712024), Funds of the Shandong "Double Tops" Program (SYL2017YSTD03) and a project (dxkt201707) from State Key Laboratory of Crop Biology. The funding bodies had no role in the design of this study, the data analysis and interpretation, the writing of the manuscript or the decision to publish.

\section{Availability of data and materials}

All data generated or analysed during this study are included in this published article and its supplementary information files. The datasets used and/or analysed during the current study are available from the corresponding author on reasonable request. Sequencing data for RNA editing efficiency analysis has been deposited in National Center for Biotechnology Information Sequence Read Archive database (https://trace. ncbi.nlm.nih.gov/Traces/sra/), the BioProject accession number: PRJNA679100.

\section{Ethics approval and consent to participate}

Not applicable.

\section{Consent for publication}

Not applicable.

\section{Competing interests}

The authors declare that they have no competing interests.

\section{Author details}

${ }^{1}$ State Key Laboratory of Crop Biology, College of Life Sciences, Shandong Agricultural University, Taian, Shandong 271018, PR China. ${ }^{2}$ Institute of Molecular Breeding for Maize, Qilu Normal University, Jinan 250200, PR China. ${ }^{3}$ State Key Laboratory of Crop Biology, College of Plant Protection, Shandong Agricultural University, Taian, Shandong 271018, PR China.

\section{Received: 9 May 2020 Accepted: 1 December 2020}

\section{Published online: 09 December 2020}

\section{References}

1. Chen L, Li YX, Li C, Shi Y, Song Y, Zhang D, et al. Genome-wide analysis of the pentatricopeptide repeat gene family in different maize genomes and its important role in kernel development. BMC Plant Biol. 2018:18:366.

2. Lurin C, Andrés C, Aubourg S, Bellaoui M, Bitton F, Bruyère C, et al. Genomewide analysis of Arabidopsis pentatricopeptide repeat proteins reveals their essential role in organelle biogenesis. Plant Cell. 2004;16:2089-103.

3. Chen G, Zou Y, Hu J, Ding Y. Genome-wide analysis of the rice PPR gene family and their expression profiles under different stress treatments. BMC Genomics. 2018;19:720

4. Schmitz-Linneweber C, Small I. Pentatricopeptide repeat proteins: a socket set for organelle gene expression. Trends Plant Sci. 2008;13:663-70.

5. Gutmann B, Royan S, Schallenberg-Rüdinger M, Lenz H, Castleden IR, McDowell $R$, et al. The expansion and diversification of pentatricopeptide repeat RNA-editing factors in plants. Mol Plant. 2020;13:215-30.

6. Small ID, Peeters N. The PPR motif - a TPR-related motif prevalent in plant organellar proteins. Trends Biochem Sci. 2000:25:46-7.

7. Yin P, Li Q, Yan C, Liu Y, Liu J, Yu F, et al. Structural basis for the modular recognition of single-stranded RNA by PPR proteins. Nature. 2013;504:16871

8. Zhang Y, Lu C. The enigmatic roles of PPR-SMR proteins in plants. Adv Sci. 2019:6:1900361.

9. Liu S, Melonek J, Boykin LM, Small I, Howell KA. PPR-SMRs: ancient proteins with enigmatic functions. RNA Biol. 2013;10:1501-10.

10. Barkan A, Small I. Pentatricopeptide repeat proteins in plants. Annu Rev Plant Biol. 2014:65:415-42.

11. Zhang L, Zhou W, Che L, Rochaix JD, Lu C, Li W, et al. PPR protein BFA2 is essential for the accumulation of the atpH/F transcript in chloroplasts. Front Plant Sci. 2019;10:446.
12. Dudkina NV, Heinemeyer J, Sunderhaus S, Boekema EJ, Braun HP. Respiratory chain supercomplexes in the plant mitochondrial membrane. Trends Plant Sci. 2006;11:232-40.

13. Sun F, Zhang $X$, Shen $Y$, Wang $H$, Liu $R$, Wang $X$, et al. The pentatricopeptide repeat protein EMPTY PERICARP8 is required for the splicing of three mitochondrial introns and seed development in maize. Plant J. 2018;95:919-32.

14. Li XJ, Zhang YF, Hou M, Sun F, Shen Y, Xiu ZH, et al. Small kernel 1 encodes a pentatricopeptide repeat protein required for mitochondrial nad7 transcript editing and seed development in maize (Zea mays) and rice (Oryza sativa). Plant J. 2014;79:797-809.

15. Takenaka M, Zehrmann A, Verbitskiy D, Härtel B, Brennicke A. RNA editing in plants and its evolution. Annu Rev Genet. 2013;47:335-52.

16. Ichinose M, Sugita M. RNA editing and its molecular mechanism in plant organelles. Genes (Basel). 2016;8:5.

17. Xiu Z, Sun F, Shen $Y$, Zhang $X$, Jiang $R$, Bonnard G, et al. EMPTY PERICARP16 is required for mitochondrial nad2 intron 4 cis-splicing, complex I assembly and seed development in maize. Plant J. 2017;85:507-19.

18. Cai M, Li S, Sun F, Sun Q, Zhao H, Ren $X$, et al. Emp10 encodes a mitochondrial PPR protein that affects the cis-splicing of nad2 intron 1 and seed development in maize. Plant J. 2017;91:132-44.

19. Chen X, Feng F, Qi W, Xu L, Yao D, Wang Q, et al. Dek35 encodes a PPR protein that affects cis-splicing of mitochondrial nad4 intron 1 and seed development in maize. Mol Plant. 2017;10:427-41.

20. Ren RC, Wang LL, Zhang L, Zhao YJ, Wu JW, Wei YM, et al. DEK43 is a Ptype pentatricopeptide repeat (PPR) protein responsible for the Cis-splicing of nad4 in maize mitochondria. J Integr Plant Biol. 2020;62:299-313.

21. Ren RC, Lu X, Zhao YJ, Wei YM, Wang LL, Zhang L, et al. Pentatricopeptide repeat protein DEK45 is required for mitochondrial function and kernel development in maize. J Exp Bot. 2019;70:6163-79.

22. Sung TY, Tseng CC, Hsieh MH. The SLO1 PPR protein is required for RNA editing at multiple sites with similar upstream sequences in Arabidopsis mitochondria. Plant J. 2010;63:499-511.

23. Hammani K, des Francs-Small CC, Takenaka M, Tanz SK, Okuda K, Shikanai T, et al. The pentatricopeptide repeat protein OTP87 is essential for RNA editing of nad7 and atp1 transcripts in Arabidopsis mitochondria. J Biol Chem. 2011;286:21361-71.

24. Verbitskiy D, Merwe JA, Zehrmann A, Härtel B, Takenaka M. The E-class PPR protein MEF3 of Arabidopsis thaliana can also function in mitochondrial RNA editing with an additional DYW domain. Plant Cell Physiol. 2012;53:358-67.

25. Takenaka M. MEF9, an E-subclass pentatricopeptide repeat protein, is required for an RNA editing event in the nad7 transcript in mitochondria of Arabidopsis. Plant Physiol. 2010;152:939-47.

26. Härtel B, Zehrmann A, Verbitskiy D, Takenaka M. The longest mitochondrial RNA editing PPR protein MEF12 in Arabidopsis thaliana requires the fulllength E domain. RNA Biol. 2013;10:1543-8.

27. Toda T, Fujii S, Noguchi K, Kazama T, Toriyama K. Rice MPR25 encodes a pentatricopeptide repeat protein and is essential for RNA editing of nad5 transcripts in mitochondria. Plant J. 2012:72:450-60.

28. Yap A, Kindgren P, Colas des Francs-Small C, Kazama T, Tanz SK, Toriyama K, et al. AEF1/MPR25 is implicated in RNA editing of plastid atpF and mitochondrial nad5, and also promotes atpF splicing in Arabidopsis and rice. Plant J. 2015:81:661-669.

29. Weißenberger S, Soll J, Carrie C. The PPR protein SLOW GROWTH 4 is involved in editing of nad 4 and affects the splicing of nad2 intron 1. Plant Mol Biol. 2017;93:355-68.

30. Wang HC, Sayyed A, Liu XY, Yang YZ, Sun F, Wang Y, et al. SMALL KERNEL4 is required for mitochondrial cox 1 transcript editing and seed development in maize. J Integr Plant Biol. 2020;62:777-92.

31. Sun F, Wang X, Bonnard G, Shen Y, Xiu Z, Li X, et al. Empty pericarp7 encodes a mitochondrial E-subgroup pentatricopeptide repeat protein that is required for ccmFN editing, mitochondrial function and seed development in maize. Plant J. 2015;84:283-95.

32. Li X, Gu W, Sun S, Chen Z, Chen J, Song W, et al. Defective kernel 39 encodes a PPR protein required for seed development in maize. J Integr Plant Biol. 2018;60:45-64.

33. Qi W, Tian Z, Lu L, Chen X, Chen X, Zhang W, et al. Editing of mitochondrial transcripts nad3 and cox2 by Dek10 is essential for mitochondrial function and maize plant development. Genetics. 2017;205:1489-501.

34. Lu X, Liu J, Ren W, Yang Q, Chai Z, Chen R, et al. Gene-indexed mutations in maize. Mol Plant. 2018;11:496-504. 
35. Bentolila S, Oh J, Hanson MR, Bukowski R. Comprehensive high-resolution analysis of the role of an Arabidopsis gene family in RNA editing. PLoS Genet. 2013;9:e1003584.

36. Clifton SW, Minx P, Fauron CM, Gibson M, Allen JO, Sun H, et al. Sequence and comparative analysis of the maize NB mitochondrial genome. Plant Physiol. 2004;136:3486-503.

37. Xiao H, Zhang Q, Qin X, Xu Y, Ni C, Huang J, et al. Rice PPS1 encodes a DYW motif-containing pentatricopeptide repeat protein required for five consecutive RNA-editing sites of nad3 in mitochondria. New Phytol. 2018;220:878-92.

38. Xie T, Chen D, Wu J, Huang X, Wang Y, Tang K, et al. Growing slowly 1 locus encodes a PLS-type PPR protein required for RNA editing and plant development in Arabidopsis. J Exp Bot. 2016;67:5687-98.

39. Li XL, Huang WL, Yang HH, Jiang RC, Sun F, Wang HC, et al. EMP18 functions in mitochondrial atp6 and cox2 transcript editing and is essential to seed development in maize. New Phytol. 2019;221:896-907.

40. Vanlerberghe GC. Alternative oxidase: a mitochondrial respiratory pathway to maintain metabolic and signaling homeostasis during abiotic and biotic stress in plants. Int J Mol Sci. 2013;14:6805-47.

41. Kühn K, Yin G, Duncan O, Law SR, Kubiszewski-Jakubiak S, Kaur P, et al. Decreasing electron flux through the cytochrome and/or alternative respiratory pathways triggers common and distinct cellular responses dependent on growth conditions. Plant Physiol. 2015;167:228-50.

42. Glass F, Härtel B, Zehrmann A, Verbitskiy D, Takenaka M. MEF13 requires MORF3 and MORF8 for RNA editing at eight targets in mitochondrial mRNAs in Arabidopsis thaliana. Mol Plant. 2015;8:1466-77.

43. Takenaka M, Zehrmann A, Verbitskiy D, Kugelmann M, Härtel B, Brennicke A. Multiple organellar RNA editing factor (MORF) family proteins are required for RNA editing in mitochondria and plastids of plants. Proc Natl Acad Sci U S A. 2012;109:5104-9.

44. Ren X, Pan Z, Zhao H, Zhao J, Cai M, Li J, et al. EMPTY PERICARP11 serves as a factor for splicing of mitochondrial nad 1 intron and is required to ensure proper seed development in maize. J Exp Bot. 2017:68:4571-81.

45. Qi W, Yang Y, Feng X, Zhang M, Song R. Mitochondrial function and maize kernel development requires Dek2, a pentatricopeptide repeat protein involved in nad1 mRNA splicing. Genetics. 2017;205:239-49.

46. Okuda K, Myouga F, Motohashi R, Shinozaki K, Shikanai T. Conserved domain structure of pentatricopeptide repeat proteins involved in chloroplast RNA editing. Proc Natl Acad Sci U S A. 2007;104:8178-83.

47. Yang YZ, Ding S, Wang HC, Sun F, Huang WL, Song S, et al. The pentatricopeptide repeat protein EMP9 is required for mitochondrial ccmB and rps4 transcript editing, mitochondrial complex biogenesis and seed development in maize. New Phytol. 2017;214:782-95.

48. Andrés-Colás N, Zhu Q, Takenaka M, De Rybel B, Weijers D, Van Der Straeten D. Multiple PPR protein interactions are involved in the RNA editing system in Arabidopsis mitochondria and plastids. Proc Natl Acad Sci U S A. 2017;114:8883.

49. Diaz MF, Bentolila S, Hayes ML, Hanson MR, Mulligan RM. A protein with an unusually short PPR domain, MEF8, affects editing at over 60 Arabidopsis mitochondrial C targets of RNA editing. Plant J. 2017;92:638-49.

50. Guillaumot D, Lopez-Obando M, Baudry K, Avon A, Rigaill G, Falcon de Longevialle A, et al. Two interacting PPR proteins are major Arabidopsis editing factors in plastid and mitochondria. Proc Natl Acad Sci U S A. 2017; 114:8877-82.

51. Wang Y, Liu XY, Yang YZ, Huang J, Sun F, Lin J, et al. Empty Pericarp21 encodes a novel PPR-DYW protein that is required for mitochondrial RNA editing at multiple sites, complexes I and V biogenesis, and seed development in maize. PLoS Genet. 2019;15:e1008305.

52. Dai D, Jin L, Huo Z, Yan S, Ma Z, Qi W, et al. Maize pentatricopeptide repeat protein DEK53 is required for mitochondrial RNA editing at multiple sites and seed development. J Exp Bot. 2020;71:6246-61.

53. Chateigner-Boutin AL, des Francs-Small CC, Delannoy E, Kahlau S, Tanz SK, de Longevialle AF, et al. OTP70 is a pentatricopeptide repeat protein of the E subgroup involved in splicing of the plastid transcript rpoC1. Plant J. 2011; 65:532-42.

54. Castandet B, Choury D, Bégu D, Jordana X, Araya A. Intron RNA editing is essential for splicing in plant mitochondria. Nucleic Acids Res. 2010;38:711221.

55. Farré JC, Aknin C, Araya A, Castandet B. RNA editing in mitochondrial transintrons is required for splicing. PLoS One. 2012;7:e52644.

56. Xu C, Song S, Yang YZ, Lu F, Zhang MD, Sun F, et al. DEK46 performs C-to-U editing of a specific site in mitochondrial nad7 introns that is critical for intron splicing and seed development in maize. Plant J. 2020;103:1767-82.
57. Keren I, Tal L, des Francs-Small CC, Araújo WL, Shevtsov S, Shaya F, et al. nMAT1, a nuclear-encoded maturase involved in the trans-splicing of nad1 intron 1, is essential for mitochondrial complex I assembly and function. Plant J. 2012;71:413-26.

58. Brangeon J, Sabar M, Gutierres S, Combettes B, Bove J, Gendy C, et al. Defective splicing of the first nad4 intron is associated with lack of several complex I subunits in the Nicotiana sylvestris NMS1 nuclear mutant. Plant J. 2000;21:269-80.

59. Ren Z, Fan K, Fang T, Zhang J, Yang L, Wang J, et al. Maize empty pericarp602 encodes a P-type PPR protein that is essential for seed development. Plant Cell Physiol. 2019;60:1734-46.

60. Pan Z, Ren X, Zhao H, Liu L, Tan Z, Qiu F. A mitochondrial transcription termination factor, ZmSmk3, is required for nad1 intron4 and nad4 intron1 splicing and kernel development in maize. G3 (Bethesda). 2019;9:2677-86.

61. Chen Z, Wang HC, Shen J, Sun F, Wang M, Xu C, et al. PPR-SMR1 is required for the splicing of multiple mitochondrial introns, interacts with Zm-mCSF1, and is essential for seed development in maize. J Exp Bot. 2019;70:5245-58.

62. Jiao Y, Peluso P, Shi J, Liang T, Stitzer MC, Wang B, et al. Improved maize reference genome with single-molecule technologies. Nature. 2017;546: $524-7$.

63. Liu YJ, Xiu ZH, Meeley R, Tan BC. Empty pericarp5 encodes a pentatricopeptide repeat protein that is required for mitochondrial RNA editing and seed development in maize. Plant Cell. 2013;25:868-83.

64. Yoo SD, Cho YH, Sheen J. Arabidopsis mesophyll protoplasts: a versatile cell system for transient gene expression analysis. Nat Protoc. 2007;2:1565-72.

\section{Publisher's Note}

Springer Nature remains neutral with regard to jurisdictional claims in published maps and institutional affiliations.
Ready to submit your research? Choose BMC and benefit from:

- fast, convenient online submission

- thorough peer review by experienced researchers in your field

- rapid publication on acceptance

- support for research data, including large and complex data types

- gold Open Access which fosters wider collaboration and increased citations

- maximum visibility for your research: over $100 \mathrm{M}$ website views per year

At BMC, research is always in progress.

Learn more biomedcentral.com/submissions 